\title{
Hysteretic Percolation from Locally Optimal Individual Decisions
}

\author{
Malte Schröder, ${ }^{1,2 *}$ Jan Nagler, ${ }^{3,4, \dagger}$ Marc Timme, ${ }^{1,2, \ddagger}$ and Dirk Witthaut ${ }^{5,6,8}$ \\ ${ }^{1}$ Chair for Network Dynamics, Center for Advancing Electronics Dresden (cfaed) and Institute for Theoretical Physics, \\ Technical University of Dresden, 01069 Dresden, Germany \\ ${ }^{2}$ Network Dynamics, Max Planck Institute for Dynamics and Self-Organization (MPIDS), 37077 Göttingen, Germany \\ ${ }^{3}$ ETH Zürich, Wolfgang-Pauli-Strasse 27, CH-8093 Zürich, Switzerland \\ ${ }^{4}$ Computational Social Science, Department of Humanities, Social and Political Sciences, ETH Zurich, \\ Clausiusstrasse 50, CH-8092 Zurich, Switzerland \\ ${ }^{5}$ Forschungszentrum Jülich, Institute for Energy and Climate Research-Systems Analysis and Technology Evaluation (IEK-STE), \\ 52428 Jülich, Germany \\ ${ }^{6}$ Institute for Theoretical Physics, University of Cologne, 50937 Köln, Germany
}

(Received 15 September 2017; revised manuscript received 10 April 2018; published 14 June 2018)

\begin{abstract}
The emergence of large-scale connectivity underlies the proper functioning of many networked systems, ranging from social networks and technological infrastructure to global trade networks. Percolation theory characterizes network formation following stochastic local rules, while optimization models of network formation assume a single controlling authority or one global objective function. In socioeconomic networks, however, network formation is often driven by individual, locally optimal decisions. How such decisions impact connectivity is only poorly understood to date. Here, we study how large-scale connectivity emerges from decisions made by rational agents that individually minimize costs for satisfying their demand. We establish that the solution of the resulting nonlinear optimization model is exactly given by the final state of a local percolation process. This allows us to systematically analyze how locally optimal decisions on the microlevel define the structure of networks on the macroscopic scale.
\end{abstract}

DOI: 10.1103/PhysRevLett.120.248302

The proper functioning of networked systems fundamentally relies on their established large-scale connectivity. The global connectivity of social, economic, and technological networks, such as the internet, trade, and transportation networks, enables global communication and exchange, but also the rapid spreading of diseases [1-7]. The loss of connectivity, or even of a single connection, may cause catastrophic effects such as the collapse of ecological networks, blackouts of power grids and other infrastructures, or even a global economic crisis [8-15]. Understanding how global connectivity emerges thus constitutes a key challenge in the field of network science.

Two major theoretical approaches have been established for revealing core properties of the emergence of large-scale connectivity. Firstly, the theory of percolation provides fundamental insights about network formation processes by assuming that new links are established stochastically according to some local rule [16,17]. For such percolation models, a variety of distinct structure-forming phenomena have been observed, where diverse network topologies emerge even for simple link formation rules [7,18-24]. Secondly, global optimization models explain network formation controlled by a central authority or driven by a single global objective function. These models have been studied to construct and understand aspects of the structure of various man-made and biological networks [25-32].
The formation of many socioeconomic networks, however, is driven by local agents making individual decisions based on optimizing their own goals. Such settings result in networks constrained by many individual, yet interacting optimization problems. A similar motivation underlies game-theoretic models of network formation [33-41]. These models allow a more detailed analysis of the formation process and the stability of the resulting network. Unsurprisingly, however, they are often hard or even impossible to solve, especially for larger networks, thus limiting mechanistic insights.

In this Letter we study network formation processes based on rational agents that individually optimize their own local objective function. Given costs for production and transaction (including transport) in an underlying transport network, each agent satisfies its own demand at minimal costs $[42,43]$. We establish an exact mapping between the solutions of the resulting nonlinear optimization problems and the states of a local percolation process. This enables us to systematically investigate optimal collective network formation and to reveal discontinuous transitions of the network structure and hysteresis. These effects are independent of the network topology or specific choices of the cost functions. The proposed framework thus bridges global (deterministic) network optimization and stochastic local percolation. 
From optimization to percolation.-We analyze a network formation model based on the following fundamental network supply problem (Fig. 1). Consider an underlying network of $N$ nodes and $M$ links, describing agents and potential transportation routes, where each agent must satisfy its demand. We study the network of trades that evolves between the nodes, similar to bond percolation on an underlying network or random graph [16].

Specifically, we assume each node $i \in\{1, \ldots, N\}$ is an agent with a fixed demand $D_{i}$. The agent satisfies this demand by purchasing supplies $S_{k i} \geq 0$ from any nodes $k$, including itself, under the constraint that $\sum_{k} S_{k i}=D_{i}$. (Throughout the Letter sums run over all nodes, here $k \in\{1, \ldots, N\}$, unless otherwise noted.) Each agent tries to achieve this with minimal cost

$$
K_{i}=\sum_{k} K_{k i}^{P}+K_{k i}^{T}
$$

including both production costs $K_{k i}^{P}$ at node $k$ as well as transaction costs $K_{k i}^{T}$ between the two nodes (see Fig. 1).

The production costs depend nonlinearly on the purchases $S_{k i}$

$$
K_{k i}^{P}=p_{k}\left(S_{k}\right) S_{k i},
$$

since the costs per unit $p_{k}\left(S_{k}\right)$ typically depend on the total production $S_{k}=\sum_{j} S_{k j}$ at node $k$. Often, the production costs per unit are decreasing, $d p_{k} / d S_{k}<0$, accounting for, e.g., increased efficiency with increased production, commonly referred to as economies of scale.

The transaction costs are proportional to the amount of transported goods $S_{k i}$ and to the effective distance $T_{k i}$ between the two nodes,

$$
K_{k i}^{T}=p_{T} S_{k i} T_{k i},
$$

where $T_{k i}=\sum_{e} t_{e}$ is given as the sum of the distances $t_{e}$ of all edges $e$ along the (shortest) path between $k$ and $i$ in the underlying transport network. The factor $p_{T}$ denotes the transaction costs per unit good and unit distance, describing effects of fuel costs or delivery times.

All agents solve their individual nonlinear optimization problem [Eq. (1)] simultaneously, defining the network of optimal purchases $S_{k i}$. The resulting state of this network then corresponds to a Nash equilibrium [44], where no agent can reduce its cost by changing its purchases given that all other purchases remain constant.

Results.-A simple, yet efficient solution to this problem can be found for nonincreasing production cost per unit $p_{k}$ : in this case any agent $i$ chooses a single supplier $i^{*}$, such that $S_{i^{*} i}=D_{i}$ and $S_{k i}=0$ for $k \neq i^{*}$. In general each agent would have to check each node in the network to find its optimal supplier. Interestingly, if the demand of all agents is identical, $D_{i} \equiv D$, this optimal supplier can be found locally: An agent $i$ just has to query its direct neighbors about their current suppliers to find its optimal supplier $i^{*}$.

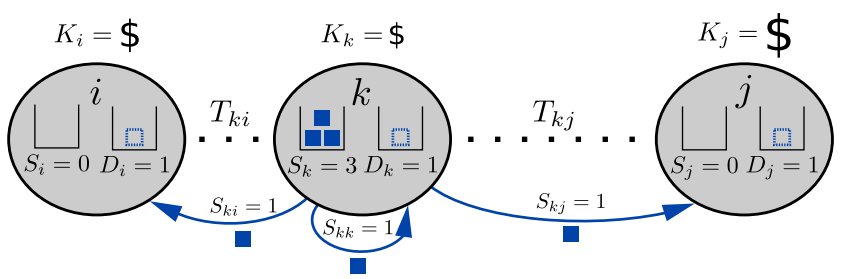

FIG. 1. Network supply problem. Each agent $i$ satisfies its demand $D_{i}$ by purchasing supplies $S_{k i}$ from nodes $k$ at minimum costs via the available transport routes (dashed lines). The costs $K_{i}=\sum_{k} K_{k i}^{P}+K_{k i}^{T}$ for these purchases include production costs $K_{k i}^{P}$ at node $k$ and transaction costs $K_{k i}^{T}$ between the nodes [Eq. (1)]. The production costs depend on the total production $S_{k}=\sum_{i} S_{k i}$ at node $k$ [Eq. (2)]. The transaction costs are proportional to the effective distance $T_{k i}$ of the transport route between the nodes [Eq. (3)].

Here, we provide a brief intuitive argument: Any purchase of agent $i$ has to be transported via one of its neighbors $j$. Since transaction costs are additive over the transport links and all agents have identical demand, agent $j$ effectively solves the same optimization problem as agent $i$ (minus the transaction costs from $j$ to $i$ ). When $j$ finds its optimal supplier, this supplier is also a potential optimal supplier of agent $i$ when transporting via $j$. Thus, agent $i$ simply compares the suppliers of all its neighbors (all potentially optimal suppliers, one for each possible path of transport). One of these must then be the optimal supplier for agent $i$ (see Supplemental Material Secs. I and II for a rigorous proof and details of the simulation [45]).

We investigate this local percolation model starting with large transaction costs, $p_{T}=\infty$, and, correspondingly, only internal production $i^{*}=i$ and $S_{i i}=D_{i}$. As $p_{T}$ decreases, the transaction costs decrease and agents minimize their total costs by establishing external purchases $S_{k i}$ from nodes with lower production costs. Finally, transaction costs disappear at $p_{T}=0$ and all agents will have the same supplier minimizing their production costs (Fig. 2). We study the size $C\left(i^{*}\right)$ of the connected components (clusters) in the network defined by these purchases, this means the number of agents $\left\{i_{1}, i_{2}, \ldots\right\}$ with the same supplier $i^{*}$. As for standard percolation we record the size $C_{1}\left(p_{T}\right)$ of the currently largest cluster (and $C_{2}$ for the second largest cluster and so on). In the following examples we consider linearly decreasing production costs per unit $p_{k}\left(S_{k}\right)=b_{k}-a S_{k}$, where $a \geq 0$ directly quantifies the strength of the economies of scale. The results are qualitatively unchanged for all forms of decreasing $p_{k}$ (see Supplemental Material [45]).

Discontinuous percolation and hysteresis.-We illustrate the emergence of connectivity in a random spatially embedded network in Figs. 3(a) and 3(b), revealing the importance of economies of scale. Weak economies of scale (small $a$ ) lead to a continuous growth of the largest cluster. Sufficiently strong economies of scale lead to a discontinuous evolution of the size of the largest cluster in 


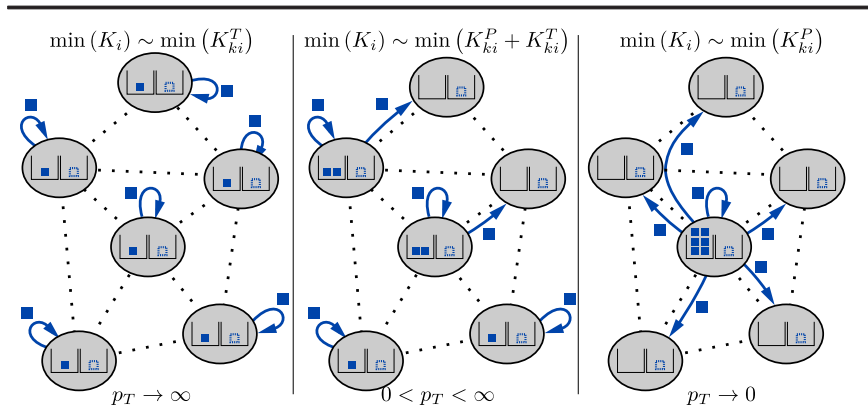

FIG. 2. Local percolation induced by optimization. Every agent chooses a single optimal supplier to satisfy its demand if the production costs per unit $p_{k}\left(S_{k}\right)$ decrease with increasing production (economies of scale). When the transaction costs per unit $p_{T}$ are large, all agents make only local purchases from their own node. As $p_{T}$ decreases, the transaction costs decrease and agents start to purchase from other nodes. Some potential transportation routes (dashed lines) become active trade links (arrows). This trade network grows along the shortest paths in the network as transaction costs become smaller. As the transaction costs disappear $\left(p_{T} \rightarrow 0\right)$, the network will become globally connected. All agents then share the same supplier, minimizing their production costs. If the demand of all agents is identical $D_{i} \equiv D$, the solution to the supply problem can be found locally and is given by the final state of a local percolation model.

the network. A microscopic decrease of the transaction costs triggers a cascade of decisions: As the cluster size increases, the production costs of its supplier decrease and a large fraction of agents join this connected component. In the language of percolation, a giant connected component emerges in a continuous (weak economies of scale) or discontinuous (strong economies of scale) phase transition.

Moreover, multiple stable states exist for sufficiently strong economies of scale. In an intermediate interval of transaction costs $p_{T}$ the network settles on one of the possible structures, depending on the previous state of the network: hysteresis emerges. Thus, a large cluster may remain stable after it has emerged for decreasing $p_{T}$, even when $p_{T}$ is increased again [Figs. 3(b) and 3(c)].

Underlying mechanism.-To understand the mechanism underlying these different transitions, we analyze a mathematically tractable system in detail. We consider a network of all-to-all coupled units with demand $D_{i}=1 / \mathrm{N}$ separated by effective distances $t_{e}=1$ for all edges $e$. We take $b_{i}=i / N$ for $i \in\{1, \ldots, N\}$, approximating the uniform distribution $b_{i} \in[0,1]$ in the limit of large system size $N$.

We now track individual decisions by considering the cost per unit $K_{i}(k)$ agent $i$ pays for purchases at node $k$. Since transaction costs across all links are identical, the first link to be established will be between the node with the highest production cost (node $N$ ) and the one with the smallest (node 1). This happens when the cost per unit $K_{N}(1)$ for agent $N$ to import from node 1 become smaller than the cost $K_{N}(N)$ to buy internally: $K_{N}(1)=$ $1 / N-2 a / N+p_{T}<1-a / N=K_{N}(N)$, that is for $p_{T}<p_{T}^{N}=1-1 / N+a / N$. Similarly, we can calculate when the next link between agent $N-1$ and node 1 is established: $\quad K_{N-1}(1)=1 / N-3 a / N+p_{T}<(N-1) / N-$ $a / N=K_{N-1}(N-1)$, that is for $p_{T}<p_{T}^{N-1}=1-2 / N+$ $2 a / N$. The other agents follow the same pattern.

Considering the two links, we now have to distinguish two cases: if $a<a_{c}=1$, then $p_{T}^{N-1}<p_{T}^{N}$ and the agents $N$ and $N-1$ will establish their links sequentially at different values of $p_{T}$. The largest cluster will grow continuously with a slope of $(1 / N) /\left(p_{T}^{N-1}-p_{T}^{N}\right)=1 /(a-1)$. However, if the economies of scale are stronger $\left(a \geq a_{c}=1\right)$, the cost at node 1 decrease sufficiently for the next link to be established immediately since $p_{T}^{N-1} \geq p_{T}^{N}$. The cluster grows discontinuously in a single cascade. If the economies of scale are even stronger $\left(a>a_{c}=1\right)$, the cluster is stable with respect to single (a)

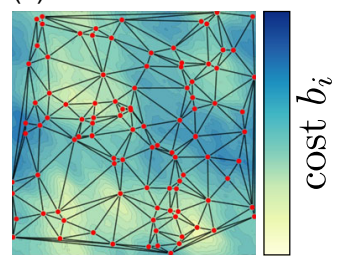

(b)

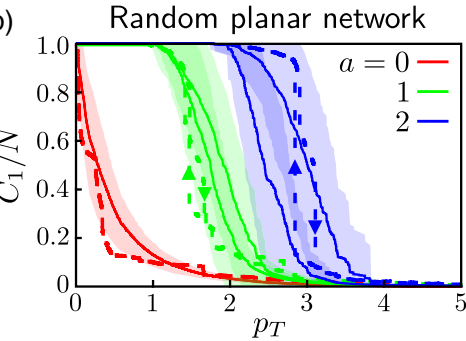

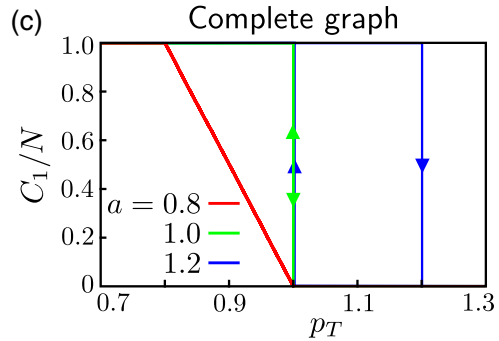

FIG. 3. From continuous to discontinuous transitions and hysteresis. The type of the transition to a single globally connected component changes depending on the strength of the economies of scale in the cost function. (a) Example of a small random network embedded in the unit square. The parameters $b_{i} \in[0,1]$ are given by the real part of a smooth random function $b(x, y)$ generated from $2^{10} \times 2^{10}$ discrete Fourier modes with mean amplitude 0 and variance $S\left(\omega_{x}, \omega_{y}\right)=\left(\omega_{x}^{2}+\omega_{y}^{2}\right)^{-2}$ (see also Supplemental Material Sec. III [45]). (b) Single realizations (dashed lines) of the evolution of the relative size of the largest cluster for a random planar network with $N=10^{4}$ nodes and average and standard deviation (solid lines and shading) over 100 random realizations of the $b_{i}$. (c) The predicted behavior for a completely connected network in the mean field limit $N \rightarrow \infty$ (see text). Weak economies of scale $\left(a<a_{c}\right)$ lead to a continuous growth of the largest cluster. Sufficiently strong economies of scale $\left(a>a_{c}\right)$ lead to a discontinuous transition. Reversing the process, i.e., increasing the transaction costs, leads to a direct reversal for weak economies of scale, but hysteresis is observed for strong economies of scale. 

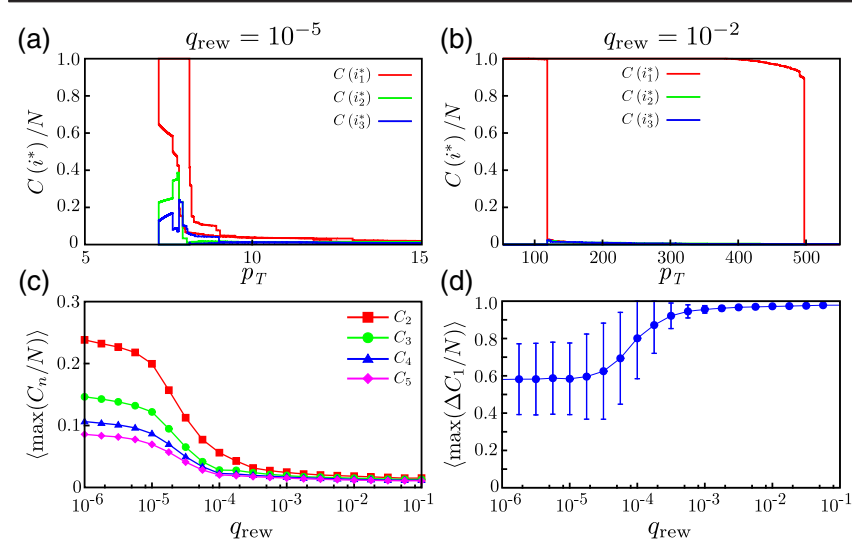

FIG. 4. Impact of the network structure on the percolation transition. Size and discontinuities of large connected components for an underlying Watts-Strogatz small world network $\left(N=10^{4}, k=8\right.$, see Supplemental Material Sec. III and Sec. V [45]). (a),(b) Single realizations for the size of the components $C\left(i^{*}\right)$ of three specific large suppliers. In a network with large diameter [panel (a), $q_{\text {rew }}=10^{-5}$ ] multiple clusters grow simultaneously and merge for small $p_{T}$. In a network with small diameter [panel (b), $q_{\text {rew }}=10^{-2}$ ] one large cluster emerges in a single cascade. (c) Maximum size of the $n$-th largest cluster as a function of the topological randomness $q_{\text {rew }}$ (error bars omitted for visibility). (d) Largest change of the size of the largest cluster (error bars indicate the standard deviation). Averages are taken over 100 realizations.

agents changing their supplier for larger values of $p_{T}$, causing hysteresis when increasing $p_{T}$. This qualitative behavior is independent of the network topology in the sense that for sufficiently large economies of scale the transition will always become discontinuous (see Supplemetal Material [45]).

Impact of network topology.-Besides changes in production and transaction costs, the growth of the trade network is also determined by changes of the transport routes, that means by the underlying physical transportation network. For the same economies of scale we find different routes of network formation depending on the structure of the network. If the network diameter (the longest shortest path between any two nodes) is small, the paths in the network are short and only one cluster emerges. If the diameter is large, multiple large clusters appear. This difference is already evident when comparing the spatially embedded (large diameter) and complete network (small diameter) for $a=1$ [compare Figs. 3(b) and 3(c)].

To systematically study this effect we fix the economies of scale $a=1$ and consider a network class introduced by Watts and Strogatz [23]. Starting from a regular ring network with a large diameter where each node is connected to its $k$ neighbors, each link is randomly rewired with probability $q_{\text {rew }}$. This introduces shortcuts and reduces the diameter of the network. If the diameter is large $\left[q_{\text {rew }}\right.$ small, Fig. 4(a)], different suppliers can attract large clusters of agents from their local part of the network when $p_{T}$ decreases. Fig. 4(c) illustrates the maximum size of the $n$-th largest cluster, showing that multiple large clusters emerge for $q_{\text {rew }} \leq 10^{-5}$ (less than one shortcut per node). Only for small values of $p_{T}$ do these clusters interact and finally merge in small cascades to a single giant cluster. If the diameter is small $\left[q_{\text {rew }}\right.$ large, Fig. 4(b) $]$, only a single cluster emerges, attracting nodes from all parts of the
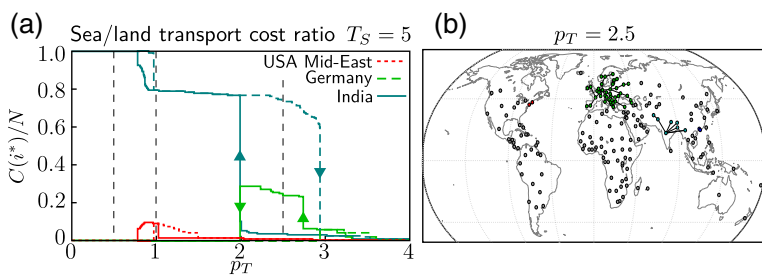

(c)

$p_{T}=1.0$

(d)

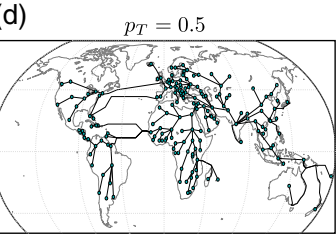

(e) Sea/land transport cost ratio $T_{S}=0.2 \quad$ (f) (g)
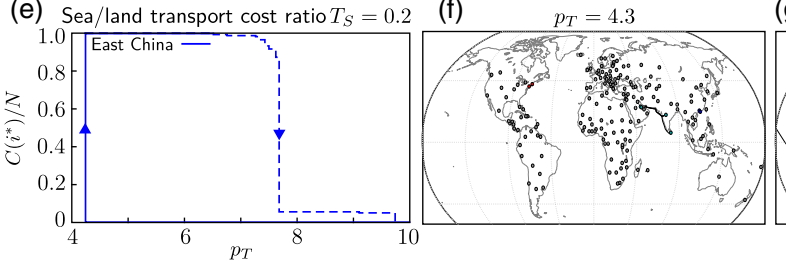

(g) $\quad p_{T}=4.2$

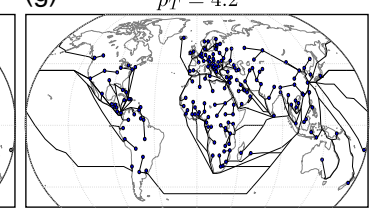

FIG. 5. Preferred modes of transport change network evolution. Global connectivity induced by locally optimal decisions in a model of a world transport network (see Supplemental Material Sec. VII for details [45]). (a) Evolution of the size $C\left(i^{*}\right)$ of specific clusters identified by a supplier $i^{*}$ when transport via sea is more expensive than transport via land (by a factor $T_{S}=5$ ). Because of the high costs of sea transport, the network diameter is large and multiple large clusters can emerge in different parts of the world. (b)-(d) Network structure and active trade links for different values of the transactions costs $p_{T}$. Land routes are preferred to transport via sea. (e) Evolution of the size of the emerging cluster for small sea transport costs $\left(T_{S}=0.2\right)$. Because of sea routes connecting most countries cheaply, the network diameter is small and only one large component emerges in a single large cascade. This transition happens for larger $p_{T}$ as the overall diameter of the network is much smaller. (f),(g) The state of the network immediately before and after the transition. 
network. The largest cluster then grows in a single cascade until it fills the entire network [Figs. 4(c) and 4(d)].

To illustrate this effect with a realistic network topology, we consider an elementary model of a world transport network (Fig. 5). The nodes of the network represent individual countries or regions and links represent transport routes via land between neighboring countries or shipping routes via sea (for a detailed description of the model parameters and all data see Supplemental Material Sec. VII [45]). Similarly to the small-world network, we explore different network structures by varying the costs for different modes of transportation, modifying the effective distances of transport via land and sea. If transport via sea is expensive, the network has a large diameter, similar to the random planar network [Figs. 3(a) and 3(b)]. Multiple large clusters appear in different regions of the world and merge when $p_{T}$ becomes small [Figs. 5(a)-5(d)]. Conversely, if transport via sea is cheap, the network becomes densely connected with a small diameter, similar to the complete graph [Fig. 3(c)], and a single largest cluster grows in a sudden cascade [Figs. 5(e)-5(g)].

Discussion.-Taken together, we have proposed a class of network formation models that demonstrates how fundamental aspects of local economic decisions impact global network formation. In contrast to random link addition extensively studied before [16,22,47], link addition in this model is driven by individual decisions: each node minimizes its own costs to satisfy a fixed demand by establishing a trade network across a given network of (potential) transport routes. The model class is general in the sense that the illustrated phenomena are independent of both the details of the network topology as well as of the details of the cost functions. Specifically, whereas we have analyzed the model with linearly decreasing production costs per unit, linearity is not required. Any decreasing cost function yields qualitatively the same results (see Supplemental Material [45]).

In an analytically solvable limit we showed that the solution of the resulting interacting optimization problems exactly maps to the final state of a local percolation process, thereby enabling a systematic analysis of the transition from localized trading to a macroscopically connected trade network. Importantly, the network evolution exhibits hysteresis, such that decreasing an external factor (e.g., the transaction cost per unit) may induce global interactions but increasing the same factor does not immediately reverse this system-scale impact.

These results directly link deterministic, optimizationbased approaches of network formation [26-30,32-35, 38-41] and prototypical percolation processes [7,18-24] based on purely random link addition. Specifically, the model illustrates the connection of (discontinuous) percolation to geographically distributed trade networks $[42,43]$. More generally, our framework shows how economic factors and the actual connectivity may shape the structure of socioeconomic networks through individual locally optimal decisions.

We thank J. Többen and S. Klipp for helpful discussions. We gratefully acknowledge support from the Göttingen Graduate School for Neurosciences and Molecular Biosciences (DFG Grant No. GSC 226/2), from the Helmholtz Association (Grant No. VH-NG-1025), the Bundesministerium für Bildung und Forschung (BMBF Grant No. 03SF0472A-F), the German Science Foundation (DFG) by a grant towards the Cluster of Excellence Center for Advancing Electronics Dresden (cfaed), the ETH Risk Center (RC SP 08-15), and SNF Grant The Anatomy of Systemic Financial Risk, No. 162776.

*malte.schroeder@tu-dresden.de

†jnagler@ethz.ch

marc.timme@tu-dresden.de

d.witthaut@fz-juelich.de

[1] R. Albert, H. Jeong, and A.-L. Barabási, Nature (London) 406, 378 (2000).

[2] R. Pastor-Satorras and A. Vespignani, Phys. Rev. Lett. 86, 3200 (2001).

[3] L. Hufnagel, D. Brockmann, and T. Geisel, Proc. Natl. Acad. Sci. U.S.A. 101, 15124 (2004).

[4] D. Brockmann, L. Hufnagel, and T. Geisel, Nature (London) 439, 462 (2006).

[5] D. Brockmann and D. Helbing, Science 342, 1337 (2013).

[6] R. Albert and A.-L. Barabási, Rev. Mod. Phys. 74, 47 (2002).

[7] M. Newman, SIAM Rev. 45, 167 (2003).

[8] R. V. Sole and M. Montoya, Proc. R. Soc. B 268, 2039 (2001).

[9] F. Schweitzer, G. Fagiolo, D. Sornette, F. Vega-Redondo, A. Vespignani, and D. R. White, Science 325, 422 (2009).

[10] S. Buldyrev, R. Parshani, G. Paul, H. E. Stanley, and S. Havlin, Nature (London) 464, 1025 (2010).

[11] S. Havlin, D. Y. Kenett, E. Ben-Jacob, A. Bunde, R. Cohen, H. Hermann, J. Kantelhardt, J. Kertész, S. Kirkpatrick, J. Kurths, J. Portugali, and S. Solomon, Eur. Phys. J. Spec. Top. 214, 273 (2012).

[12] M. Elliott, B. Golub, and M. O. Jackson, Am. Econ. Rev. 104, 3115 (2014).

[13] D. Witthaut, M. Rohden, X. Zhang, S. Hallerberg, and M. Timme, Phys. Rev. Lett. 116, 138701 (2016).

[14] H. Ronellenfitsch, D. Manik, J. Horsch, T. Brown, and D. Witthaut, IEEE Trans. Power Syst. 32, 4060 (2017).

[15] J. Nagler, A. Levina, and M. Timme, Nat. Phys. 7, 265 (2011).

[16] D. Stauffer and A. Aharony, Introduction To Percolation Theory (Taylor \& Francis, London, 1992).

[17] G. Grimmett, Percolation (Springer-Verlag, Berlin, Heidelberg, 1999).

[18] D. Achlioptas, R. D'Souza, and J. Spencer, Science 323, 1453 (2009).

[19] O. Riordan and L. Warnke, Science 333, 322 (2011).

[20] M. Schröder, S. E. Rahbari, and J. Nagler, Nat. Commun. 4, 2222 (2013). 
[21] M. Schröder, W. Chen, and J. Nagler, New J. Phys. 18, 013042 (2016).

[22] R. M. D’Souza and J. Nagler, Nat. Phys. 11, 531 (2015).

[23] D. J. Watts and S. H. Strogatz, Nature (London) 393, 440 (1998).

[24] T. Verma, F. Russmann, N. Araújo, J. Nagler, and H. Herrmann, Nat. Commun. 7, 10441 (2016).

[25] D. P. Bertsekas, Network Optimization: Continuous and Discrete Models (Athena Scientific, Belmont, 1998).

[26] M. T. Gastner and M. E. J. Newman, Phys. Rev. E 74, 016117 (2006).

[27] S. Bohn and M. O. Magnasco, Phys. Rev. Lett. 98, 088702 (2007).

[28] E. Katifori and G. J. Szöllősi, and M. O. Magnasco, Phys. Rev. Lett. 104, 048704 (2010).

[29] H. Ronellenfitsch and E. Katifori, Phys. Rev. Lett. 117, 138301 (2016).

[30] D. B. Chklovskii, T. Schikorski, and C. F. Stevens, Neuron 34, 341 (2002).

[31] R.-M. Memmesheimer and M. Timme, Physica (Amsterdam) 224D, 182 (2006).

[32] R.-M. Memmesheimer and M. Timme, Phys. Rev. Lett. 97, 188101 (2006).

[33] M. Jackson and A. Wolinsky, J. Econ. Theory 71, 44 (1996).

[34] A. Watts, Games Econ. Behav. 34, 331 (2001).

[35] M. Jackson and A. Watts, J. Econ. Theory 106, 265 (2002).

[36] M. O. Jackson, Social and Economic Networks (Princeton University Press, Princeton, 2008), Vol. 3.
[37] D. Easley and J. Kleinberg, Networks, Crowds, and Markets: Reasoning About a Highly Connected World (Cambridge University Press, Cambridge, 2010).

[38] V. Bala and S. Goyal, Econometrica 68, 1181 (2000).

[39] M. D. König, S. Battiston, M. Napoletano, and F. Schweitzer, Games Econ. Behav. 75, 694 (2012).

[40] E. Even-dar and M. Kearns, in Advances in Neural Information Processing Systems 19, edited by B. Schölkopf, J. C. Platt, and T. Hoffman (MIT Press, Cambridge, MA, 2007), pp. 385-392.

[41] O. Atabati and B. Farzad, Comput. Soc. Networks 2, 1 (2015).

[42] P. R. Krugman, Geography and Trade (MIT Press, Cambridge, MA, 1991).

[43] P. R. Krugman, J. Polit. Econ. 99, 483 (1991).

[44] M. J. Osborne and A. Rubinstein, A Course in Game Theory (MIT Press, Cambridge, MA, 1994).

[45] See Supplemental Material at http://link.aps.org/ supplemental/10.1103/PhysRevLett.120.248302, which includes Ref. [46] and contains a detailed proof of the equivalence to local percolation, additional examples, discussions of specific limiting cases, and all details on the network models as well as all data used for the world transport network.

[46] D. Saupe, The Science of Fractal Images (Springer-Verlag, Berlin, Heidelberg, 1988), pp. 71-136.

[47] B. Bollobás, Modern Graph Theory (Springer-Verlag, Berlin, Heidelberg, 1998), pp. 215-252. 